Luque, Víctor; Luque, Miguel Ángel, A vueltas con la SICAV. Reflexiones críticas desde el punto de vista económico-tributario.

\title{
A VUELTAS CON LAS SICAV. REFLEXIONES CRÍTICAS DESDE EL PUNTO DE VISTA ECONÓMICO-TRIBUTARIO
}

\author{
BACK TO SICAV. \\ CRITICAL REFLECTION FROM AN ECONOMIC-TRIBUTARY \\ POINT OF VIEW
}

\author{
VÍCTOR ANTONIO LUQUE \\ Universidad Complutense de Madrid \\ victorluquedeharo@gmail.com \\ MIGUEL ÁNGEL LUQUE \\ Universidad de Almería \\ mluque@ual.es
}

Fecha de recepción: diciembre de 2014

Fecha de aceptación: marzo de 2015

\begin{abstract}
RESUMEN:
Las Sociedades de Inversión de Capital Variable (SICAV) son un tipo de sociedad de inversión colectiva que viene recibiendo una "singular atención mediática" debido a que, al menos en apariencia, constituyen un instrumento para canalizar la inversión de los grandes patrimonios beneficiándose de un tipo nominal en el impuesto de sociedades de tan solo el $1 \%$. En el trabajo se analiza la evolución patrimonial y su distribución entre los diferentes componentes desde 2001 hasta 2014. Se aborda la posible existencia de fraude de ley en la utilización de esta figura, cuando se instrumentaliza por una o varias personas vinculadas, con la finalidad de obtener un ahorro tributario al margen del objeto (inversión colectiva) de la sociedad. Se analiza las repercusiones que tiene el hecho de que la competencia sobre el control tributario de las SICAV resida en la CNMV en lugar de la Agencia Estatal de Administración Tributaria. Por último, se presentan una serie de reflexiones, en relación con el posible riesgo de fuga de capitales que en ocasiones ha servido de justificación para el establecimiento de un régimen jurídico privilegiado.
\end{abstract}

Palabras clave: SICAV, Sistema tributario, Planificación fiscal, Fraude de ley.

\begin{abstract}
SICAVs are a type of investment society which are receiving "specific media attention" because, at least in appearance, they are an instrument which enables the channelling of investment by the large equities/net worths benefiting from a nominal type in corporate tax of only $1 \%$. This paper analyses the patrimonial evolution and its distribution between the different components
\end{abstract}


Luque, Víctor; Luque, Miguel Ángel, A vueltas con la SICAV. Reflexiones críticas desde el punto de vista económico-tributario.

from 2001 to 2014. The possible existence of law fraud in the use of this figure is discussed, when instrumented by one or more people associated for the purpose of obtaining a tributary saving outside the object (collective investment) of society. The repercussions of the fact that competition for tributary control of the SICAV resides in the CNMV instead of the State Tributary Administration Agency is analysed. Finally, some observations regarding the potential risk of capital flight that has sometimes served as justification for the establishment of a privileged legal regime are presented.

Key words: SICAV, Tributary system, Fiscal planning, Fraud Act.

JEL: H26, H21, K22, K34.

\section{INTRODUCCIÓN}

Las Sociedades de Inversión en Capital Variable (en adelante, SICAV) son una de las modalidades que, de acuerdo con la Ley 35/2003, de 4 de noviembre, de Instituciones de Inversión Colectiva (en adelante, LIIC), tienen como objeto la adquisición, tenencia, disfrute, armonización y enajenación de valores mobiliarios y de otros activos financieros ${ }^{1}$.

Desde hace tiempo, este tipo de sociedades vienen recibiendo una "singular atención mediática debido a que, al menos en apariencia, constituyen un instrumento para canalizar la inversión de los grandes patrimonios, que se beneficiarían así del singular régimen tributario, sin ser realmente entidades colectivas" $^{2}$. En este sentido, se ha advertido que la principal razón de la utilización de este tipo de instrumentos podría encontrarse en el hecho de que tributan al tipo nominal del $1 \%{ }^{3}$, frente al 35 o $30 \%$ al que estaban sometidas, por regla general, el resto de sociedades ( $25 \%$ con la nueva reforma fiscal $\left.{ }^{4}\right)$.

Llama la atención que, mientras que el Impuesto sobre la Renta de las Personas Físicas (en adelante IRPF) devengado en el año 2013, correspondiente a las retenciones del trabajo y de las actividades profesionales -que aglutinan más del $80 \%$ del IRPF y cuyas rentas pueden soportar tipos nominales en torno al $50 \%$ - ascendió a 65.598 millones de euros ${ }^{5}$, las SICAV contribuyeron con poco más de 23 millones de euros durante los últimos dos ejercicios. Si se tiene en cuenta que los beneficios antes de impuestos para este período fueron superiores a 3.752 millones de euros ${ }^{6}$, resulta un tipo

\footnotetext{
${ }^{1}$ Las SICAV se introdujeron en el sistema normativo español mediante el Decreto-Ley 7/1964 de Sociedades y Fondos de Inversión.

${ }^{2}$ Ramos Muñoz, D. Y Ruiz Almendral, V.: "Los problemas tributarios de las Sociedades de Inversión en Capital Variable (SICAV)", $Q F$, no octubre II, 2006, p. 10.

${ }^{3}$ Art. 29.4.a) de la Ley 27/2014, de 27 de noviembre, del Impuesto sobre Sociedades.

${ }_{5}^{4}$ Art. 29.1de la Ley 27/2014.

5 Informe Anual de Recaudación Tributaria de la AEAT 2014. Recuperado el 26 de Septiembre de 2014 (http://www.agenciatributaria.es/static_files/AEAT/Estudios/Estadisticas/Informes_Estadisticos/Informes Anuales_de_Recaudacion_Tributaria/Ejercicio_2013/IART_13.pdf)

${ }^{6}$ Fuente: CNMV (https://www.cnmv.es/DocPortal/Estadisticas/IIC/Estadisticas_IIC_2014_1T.pdf)
} 
Luque, Víctor; Luque, Miguel Ángel, A vueltas con la SICAV. Reflexiones críticas desde el punto de vista económico-tributario.

efectivo sobre resultados cercano al 0,6 \% $\%^{7}$. Si tales entidades hubieran tributado, no ya al tipo nominal del $30 \%$ en el Impuesto sobre Sociedades (en adelante, IS) que existía para las grandes empresas en 2013, sino al tipo efectivo del citado tributo sobre el resultado contable ${ }^{8}$, solo durante los años 2012 y 2013 se podrían haber ingresado en el erario público unos 370 millones de euros, cifra que equivale a más del doble de los ingresos provenientes de la reorganización y subida de las tasas judiciales (149 millones, según el Informe Anual de Recaudación Tributaria 2013), por citar un solo ejemplo.

El análisis de estos problemas es el objeto de nuestro trabajo, el cual está estructurado en cuatro partes. En primer lugar, analizaremos la evolución patrimonial de este tipo de sociedades y su distribución entre los diferentes componentes desde 2001 hasta 2014. Posteriormente, se abordará el tema de la posible existencia de fraude de ley en la utilización de esta figura. En tercer lugar, se estudiará la competencia en el control tributario de las SICAV. Seguidamente se alude a su gestión y, en quinto lugar, trataremos el posible riesgo de fuga de capitales, que en ocasiones ha servido de justificación para el establecimiento de un régimen jurídico privilegiado.

\section{EVOLUCIÓN PATRIMONIAL DE LAS SICAV}

Según los datos de la Comisión Nacional del Mercado de Valores (en adelante, CNMV) a principios de 2014 existían en España 3112 SICAV, con un patrimonio superior a 30.210 millones de euros ${ }^{9}$. Los datos estadísticos disponibles en la CNMV comienzan en $2001^{10}$. Desde esta fecha hasta 2008 , se produce un incremento considerable en el conjunto del patrimonio $(1,88 \%$ trimestral), el cual pasa de 14.120 millones de $€$ a 24.657 millones de $€$. Sin embargo, durante este período se dan dos comportamientos sustancialmente diferentes en la distribución de su patrimonio: hasta el II trimestre de 2007, todos los componentes crecen a tasas trimestrales relativamente similares (alrededor del 3,5 \%), con excepción de la tesorería que, al aumentar al 2,2 \%, pierde peso en el conjunto patrimonial. Sin embargo, a partir del III trimestre de

\footnotetext{
${ }^{7}$ Fuente: Ibídem.

${ }^{8}$ Según los datos del Informe Anual de Recaudación Tributaria de 2013, en 2012 (Cuadro 3.6) este se situó en el $10,2 \%$.

(http://www.agenciatributaria.es/static_files/AEAT/Estudios/Estadisticas/Informes_Estadisticos/Informes Anuales_de_Recaudacion_Tributaria/Ejercicio_2013/IART_13.pdf)

${ }^{9}$ Fuente: CNMV (http://www.cnmv.es/DocPortal/Estadisticas/IIC/Estadisticas_IIC_2014_2T.pdf)

${ }^{10}$ Existe una discrepancia en la estadísticas que proporciona la CNMV referentes a la distribución del patrimonio de las SIVAC en el tercer y cuarto trimestre de 2008, respecto a las que proporciona la misma entidad con una metodología anterior debido, principalmente a cambios en la valoración, que ha pasado a ser a precios de mercado y a la inclusión/exclusión de determinadas partidas en unas y otras. Por esta razón, hemos optado por realizar un estudio por separado de ambos sub-períodos. En el siguiente documento de la CNMV se explica con detalle el cambio metodológico: https://www.cnmv.es/Portal/Publicaciones/Pub_estadisticas.aspx?id=IC1

Por la misma circunstancia, no se disponen los datos del inmovilizado material e inmaterial anteriores a 2008, pero dada su aportación residual al conjunto del patrimonio, consideramos que su ausencia no desvirtúa el análisis. Igualmente, con intención de simplificar el análisis y dado que el peso de estas partidas no alcanza ni el $2 \%$ del conjunto patrimonial y que, por otra parte, su comportamiento responde a otras variables, hemos optado por no incluir la evolución de la cuenta "neto acreedores/deudores".
} 
Luque, Víctor; Luque, Miguel Ángel, A vueltas con la SICAV. Reflexiones críticas desde el punto de vista económico-tributario.

2007 (con el inicio de la crisis financiera internacional) y hasta final de 2008, se produce un cambio drástico en el comportamiento de los diferentes componentes del patrimonio de las SICAV: la cartera exterior se contrae con más intensidad $(-12,25 \%$ de tasa media de variación trimestral), mientras que la cartera interior se reduce ${ }^{11}$, pero a un ritmo mucho menor $(-1,20 \%)$. Las causas de esta disminución patrimonial se deben, tanto a la caída en la valoración de los activos incluidos en estas carteras, como a la disminución cuantitativa de éstos. Reflejo de esta reducción es la preferencia por la liquidez que se da en este sub-período y que se observa en el elevado incremento de la tesorería (15,89 \% de tasa media de variación trimestral).

El período 2009-2014 lo hemos dividido, también, en dos sub-periodos, considerando la amnistía fiscal ${ }^{12}$, que finalizó en 2012 , como un hecho relevante susceptible de atraer capitales a instrumentos de inversión como las SICAV. En el primer sub-período (Primer trimestre 2009-Final de 2012), se acentúa la evolución negativa que venía experimentando la cartera interior. Mientras tanto, la cartera exterior recupera su dinamismo $(2,59 \%$ crecimiento medio trimestral), gracias tanto a la recuperación de los principales índices internacionales ${ }^{13}$, como a una mayor preferencia por las inversiones exteriores en el conjunto de las SICAV. En el segundo sub-periodo (Primer trimestre 2013-segundo trimestre 2014), se produce un incremento en todas las partidas, si bien el comportamiento está claramente dualizado.

Tabla 1.1: Evolución de la distribución del patrimonio de las SICAV

\begin{tabular}{|c|c|c|c|c|c|c|}
\cline { 2 - 7 } \multicolumn{1}{c|}{} & $\begin{array}{c}\mathbf{2 0 0 1}(\text { II) - } \\
\mathbf{2 0 0 7} \text { (II) }\end{array}$ & $\begin{array}{c}\mathbf{2 0 0 7} \text { (III) - } \\
\mathbf{2 0 0 8} \text { (IV) }\end{array}$ & $\begin{array}{c}\mathbf{2 0 0 1} \text { (II) - } \\
\mathbf{2 0 0 8} \text { (IV) }\end{array}$ & $\begin{array}{c}\mathbf{2 0 0 9} \text { (I) - } \\
\mathbf{2 0 1 2} \text { (IV) }\end{array}$ & $\begin{array}{c}\mathbf{2 0 1 3} \text { (I) - } \\
\mathbf{2 0 1 4} \text { (II) }\end{array}$ & $\begin{array}{c}\mathbf{2 0 0 9} \text { (I) - } \\
\mathbf{2 0 1 4} \text { (II) }\end{array}$ \\
\hline Patrimonio & 3,57 & $-4,64$ & 1,88 & $-0,25$ & 4,14 & 0,93 \\
\hline $\begin{array}{c}\text { Cartera } \\
\text { Interior }\end{array}$ & 3,49 & $-1,20$ & 2,54 & $-2,07$ & 0,73 & $-1,32$ \\
\hline $\begin{array}{c}\text { Cartera } \\
\text { Exterior }\end{array}$ & 3,73 & $-12,25$ & 0,32 & 2,59 & 6,91 & 3,75 \\
\hline Tesorería & 2,20 & 15,89 & 4,80 & $-0,39$ & 8,95 & 2,08 \\
\hline
\end{tabular}

Fuente: Elaboración propia a partir de los datos de la CNMV. Tasas de variación media trimestral, en términos porcentuales.

\footnotetext{
${ }^{11}$ Se considerarán cartera interior las inversiones en instrumentos financieros realizadas en mercados regulados o sistemas multilaterales de negociación radicados en España, con independencia del domicilio social de la entidad emisora del valor o su divisa de denominación, mientras que la cartera exterior está formada por las inversiones en instrumentos financieros realizadas en mercados radicados fuera de España.

12 Aprobada por el Real Decreto-Ley 12/2012, de 30 de marzo, que permitió regularizar la situación de los evasores hasta el 30 de noviembre de 2012, a cambio de abonar el 10\% de las cantidades defraudadas, a la vez que a las empresas repatriar, vía dividendos, los fondos que tenían en paraísos fiscales con un gravamen del 8\%. Vid sobre este aspecto, Navarro Sanchís, J.: "Sombras y penumbras en la declaración tributaria especial", Medidas y procedimientos contra el fraude fiscal. Hinojosa Torralvo, J.J. (dir.) Atelier, Barcelona 2010, págs. 53-74.

${ }^{13}$ Tanto S\&P 500 como Nasdaq 100 alcanzan su valor mínimo en enero de 2009 y, desde entonces hasta final de 2012, los valores de S\&P se revalorizan un 196\%, mientras que los de Nasdaq 100 lo hacen un $225 \%$.
} 
Luque, Víctor; Luque, Miguel Ángel, A vueltas con la SICAV. Reflexiones críticas desde el punto de vista económico-tributario.

Gráfico 1.1: Distribución del patrimonio de las SICAV (2001-2014)

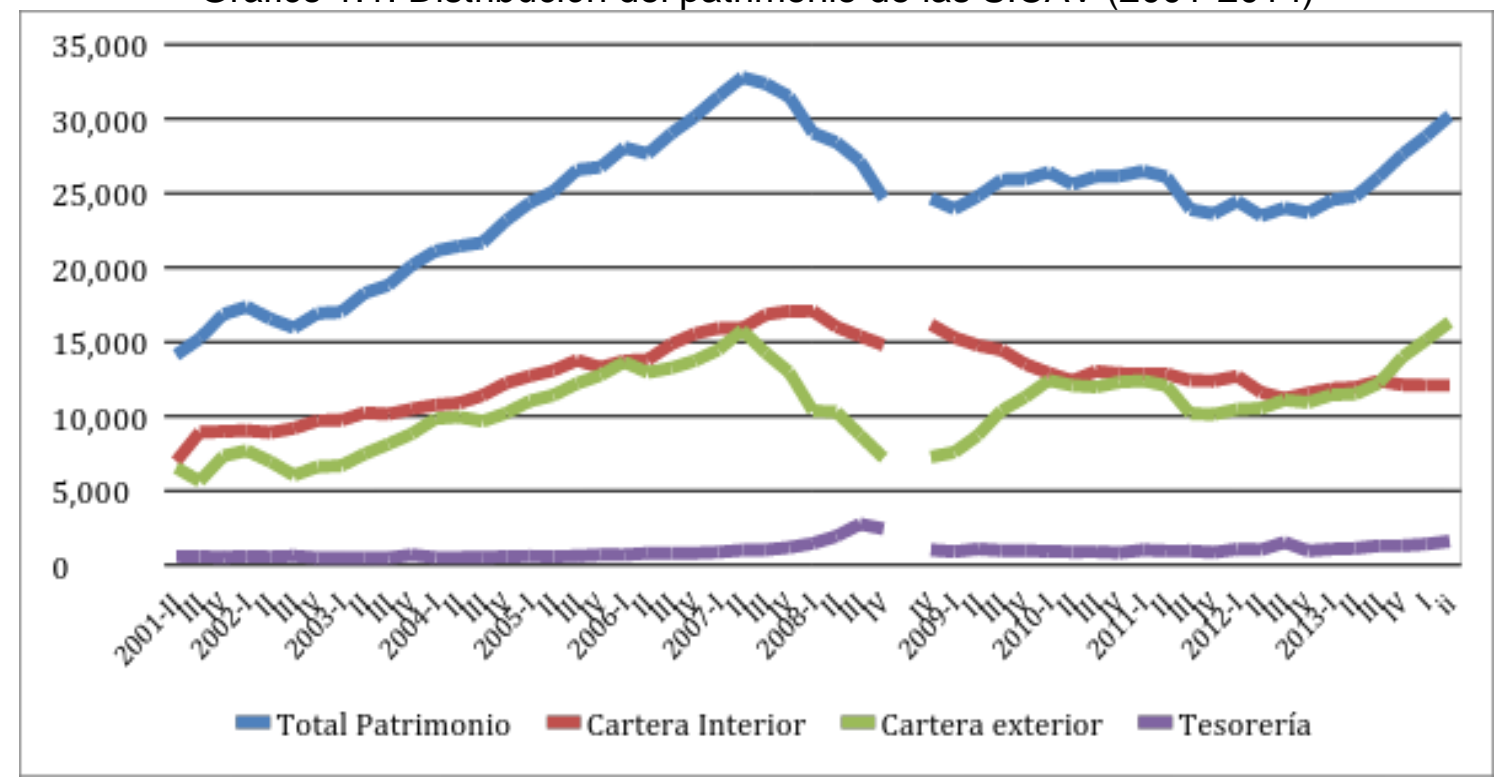

Fuente: Elaboración propia a partir de los datos de la CNMV. Valores en millones de $€$

Por un lado, la cartera exterior y la tesorería crecen a tasas medias trimestrales muy elevadas $(6,91 \%$ y $8,95 \%$ respectivamente), mientras que por el otro, la cartera interior deja de caer para pasar a tener un crecimiento reducido $(0,73 \%$ de media trimestral).

Los datos anteriores no nos permiten diferenciar en qué medida las variaciones en cada una de las carteras se debe a la caída en su valor o a la reducción cuantitativa de los activos que la integran. Para poder analizar el efecto que ha tenido la amnistía fiscal vamos a descomponer la variación patrimonial del último período en función de si ésta ha sido debida a la puesta en circulación o la recompra de antiguas acciones ${ }^{14}$, a la distribución de beneficios ${ }^{15}$, a rendimientos netos o a la revalorización de inmuebles de uso propio. Desde 2010 a 2014 la variación patrimonial en el conjunto de las SICAV ha sido de 4.285 millones de $€$; los rendimientos netos de las inversiones han alcanzado la cifra de 4.533 millones de $€^{16}$, mientras que se han recomprado acciones por valor de 162 millones de $€$. Durante este período, se repartieron sólo 85 millones de $€$ de beneficios por el conjunto de las SICAV.

Si subdividimos este período en dos, tomando como fecha divisoria el plazo en el que finalizó la amnistía fiscal (30/11/2012), podemos observar un claro cambio de tendencia a partir de esa fecha. En el primer sub-período (20102012) se produce una disminución del patrimonio de las SICAV, debido principalmente a la recompra de acciones por valor de 3.000 millones de $€$, junto con unos beneficios distribuidos de 68 millones de $€$. Ambas cifras son

\footnotetext{
${ }^{14}$ Lo cual, en la práctica, supone una desinversión en las SICAV, puesto que estas recompran sus títulos a sus propios accionistas, quedando como resultado una SICAV con menos acciones en circulación y con menor valor patrimonial.

${ }^{15}$ La distribución de beneficios entre los accionistas, como es lógico, reduce el patrimonio de la SICAV.

${ }^{16}$ Los rendimientos netos incluyen intereses, dividendos y variación de precios de los activos.
} 
Luque, Víctor; Luque, Miguel Ángel, A vueltas con la SICAV. Reflexiones críticas desde el punto de vista económico-tributario.

compensadas parcialmente con unos rendimientos netos positivos de 826 millones de $€$. En cambio, en el segundo sub-período (2013- II trimestre 2014), se observa un incremento patrimonial de 6.528 millones de $€$, los cuales vienen explicados por un aumento de la inversión en SICAV (puesta en circulación de acciones) de 2.838 millones, junto con unos rendimientos netos que alcanzaron la cifra de 3.707 millones. Los beneficios distribuidos en este último sub-periodo fueron de 17 millones de $€$.

Así, podemos concluir que, a partir de la fecha de la citada amnistía, se produjo un fuerte impulso en el crecimiento patrimonial de las SICAV basado, tanto en la revalorización de los activos, como en la puesta en circulación de nuevas acciones, a diferencia del sub-período anterior, en el que las recompras de acciones fueron superiores a la puesta en circulación y la diferencia de ambas notablemente superior a los rendimientos netos. Este crecimiento patrimonial, en lugar de haberse concentrado en la cartera de inversiones internas, se concentró, principalmente, en tesorería y cartera exterior, minimizando los posibles efectos positivos que hubiera tenido en la economía española esta entrada de capitales.

Tabla 1.2: Descomposición de la variación patrimonial

\begin{tabular}{|l|c|c|c|}
\cline { 2 - 4 } \multicolumn{1}{c|}{} & $\mathbf{2 0 1 0}$ (I)- & $\mathbf{2 0 1 2}$ (IV)- & $\mathbf{2 0 1 0}$ (I) - \\
\hline Variación del patrimonio (1+2+3+4) & $\mathbf{2 0 1 4}$ (II) & $\mathbf{2 0 1 4}$ (II) \\
\hline 1. Puesta en circulación / Recompra de acciones & -2.242 .953 & 6.528 .464 & 4.285 .511 \\
\hline 2. Beneficios brutos distribuidos & -68.451 & -16.844 & -85.295 \\
\hline 3. Rendimientos netos & 825.911 & 3.707 .262 & 4.533 .173 \\
\hline 4. Revalorización de inmuebles de uso propio & 1 & 0 & 1 \\
\hline
\end{tabular}

Fuente: Elaboración propia a partir de los datos de la CNMV. Datos en miles de $€$.

\section{EL POSIBLE FRAUDE DE LEY EN LA UTILIZACIÓN DE LAS SICAV}

El argumento que se ofrece para justificar la fiscalidad privilegiada de estas sociedades ( $1 \%$ de tipo de gravamen) se basa en el hecho de ser una clase de sociedad de inversión colectiva, de tipo abierto y libertad de acceso, con un capital inicial mínimo de 2,4 millones de euros ${ }^{17}$, dirigida a una pluralidad de ahorradores y no a intereses particulares de grupos empresariales o familiares, al exigirse un número de socios que no puede ser inferior a $100^{18}$. Ello implicaría la pérdida del control de la inversión por el socio, que pasaría a manos especializadas hasta que se transmitiera o se recibiera un dividendo,

\footnotetext{
${ }^{17}$ El art. 80.2 del Real Decreto 1082/2012, de 13 de julio, que aprueba el Reglamento de desarrollo de la Ley 35/2003, de instituciones de inversión colectiva, dispone que "el capital mínimo desembolsado de las SICAV será de 2.400 .000 euros y deberá ser mantenido mientras la sociedad figure inscrita en el registro, sin perjuicio de lo previsto en el artículo 16.1".

${ }^{18}$ Vid. art. 9.4 de la Ley 35/2003, de 4 de noviembre, de Instituciones de Inversión Colectiva. La finalidad expuesta se expresa en la propia Exposición de Motivos de la citada Ley 35/2003.
} 
Luque, Víctor; Luque, Miguel Ángel, A vueltas con la SICAV. Reflexiones críticas desde el punto de vista económico-tributario.

momento en el que tributaría por las ganancias obtenidas al tipo correspondiente en el Impuesto que grave esa renta, que en el caso del IRPF llegaría hasta el $27 \%$ (con la actual reforma tributaria, el $24 \%$ - $23 \%$ en $2016)^{19}$. Por tanto, el tipo de gravamen del $1 \%$ respondería al principio de neutralidad fiscal mediante el desplazamiento de la tributación de la sociedad al socio, para evitar la doble imposición que experimentaría la persona que opta por invertir en este tipo de entidades en lugar de hacerlo directamente en valores mobiliarios.

Las SICAV se diferencia de los fondos de inversión en que, mientras que estos se constituyen como patrimonios "sin personalidad jurídica" ${ }^{20}$, donde el participe pierde totalmente el control sobre la inversión -que queda en manos de la gestora-, en las SICAV el inversor -que suele poseer unos recursos más elevados y exigir mayor rentabilidad- adquiere la condición de accionista de una Sociedad sin limitación de participación en el capital social. Y esta circunstancia es la que ha facilitado que, en la práctica, muchas de estas entidades se utilicen como vehículo para canalizar grandes patrimonios vinculados a lazos familiares, desvirtuando su naturaleza de institución de inversión colectiva ${ }^{21}$. Como se ha afirmado, la "trampa" de estas entidades reside en el hecho de que, en determinadas ocasiones, la mayoría de las acciones pertenecen a un solo titular y funcionan, de facto, como sociedades personales, y no como sociedades abiertas a una pluralidad indeterminada de inversores ${ }^{22}$.

A este respecto, se ha denunciado en múltiples ocasiones que suele ser común que el $99 \%$ del capital social permanezca en poder de un grupo familiar y los restantes accionistas (que son empleados o familiares de empleados de la Gestora y se denominan hombres de paja o 'mariachis' y que en otros ámbitos se calificarían como testaferros ${ }^{23}$ ) posean una participación en conjunto inferior

\footnotetext{
${ }^{19}$ Para los rendimientos de más de 50.000 euros. Para los comprendidos entre 6.000 y $50.000 €$ el tipo será del $21 \%$ y para los inferiores a $6.000 €$, el $19 \%$..Vid. art. Primero, apartados cuarenta y dos, cincuenta y uno, y setenta y ocho, así como la Disposición final sexta de la Ley 26/2014, de 27 de noviembre, por el que se modifican la Ley 35/2006, de 28 de noviembre, del IRPF, el texto refundido de la Ley del Impuesto sobre la Renta de No Residentes, aprobado por el Real Decreto Legislativo 5/2004, de 5 de marzo y otras normas tributarias.

${ }^{20}$ De acuerdo con el art. 3.1 de la LIIC "Los fondos de inversión son IIC configuradas como patrimonios separados sin personalidad jurídica, pertenecientes a una pluralidad de inversores, incluidos entre ellos otras IIC, cuya gestión y representación corresponde a una sociedad gestora, que ejerce las facultades de dominio sin ser propietaria del fondo, con el concurso de un depositario, y cuyo objeto es la captación de fondos, bienes o derechos del público para gestionarlos e invertirlos en bienes, derechos, valores u otros instrumentos, financieros o no, siempre que el rendimiento del inversor se establezca en función de los resultados colectivos".

${ }^{21}$ Ramos Muñoz, D. Y Ruiz Almendral, V.: "Los problemas tributarios de las Sociedades de Inversión en Capital Variable (SICAV)", op. cit., p. 13; Tusquets Trías Bes, F.: La Sociedad de Inversión Mobiliaria de Capital Variable (SIMCAV), Marcial Pons, Madrid, 2001, p. 64.

${ }^{22}$ Martin Seco, J.F.: Economía: mentiras y trampas, Península, 2012, pp. 236 a 241.

23 De La Torre Díaz, F.: "SICAV: Una reforma necesaria", Dominio Público, 02-09-2009. http://blogs.publico.es/dominiopublico/1518/sicav-una-reforma-necesaria/. Según este autor, se les llama "mariachis", parafraseando la ranchera "con dinero y sin dinero, yo hago siempre lo que quiero y mi palabra es la ley...".
} 
Luque, Víctor; Luque, Miguel Ángel, A vueltas con la SICAV. Reflexiones críticas desde el punto de vista económico-tributario.

al $1 \%$. Es decir, personas que hacen bulto prestando su NIF, sin tener verdadera vocación inversora, con el objetivo de cumplir los requisitos formales relativos a la concurrencia de una colectividad de personas y alcanzar la cifra mínima de $100^{24}$. En este sentido, es significativo que más del $83 \%$ de las SICAV tengan menos de 150 accionistas $^{25}$.

En la medida en que su utilización, con frecuencia, está instrumentalizada por una o varias personas vinculadas a la consecución de un ahorro tributario al margen del objeto (inversión colectiva) de la Sociedad, de forma combinada con la interposición de otras sociedades holding, podría entenderse que en el caso de SICAV no colectivas nos encontraríamos ante un "fraude de ley" o conflicto en la aplicación de las normas tributarias, que implicaría una tributación al tipo general del $I^{26}$. Por este motivo, los técnicos de Hacienda han propuesto el establecimiento de un límite a la participación accionarial, para evitar el abuso que se está realizando en torno a esta figura. Como se ha afirmado, "si un solo inversor tiene el control total del fondo, este ya no es una institución de inversión colectiva y el inversor nunca pierde el control sobre su dinero", añadiendo que "en la práctica, las SICAV se utilizan exclusivamente para la gestión individual de carteras de grandes patrimonios" ${ }^{27}$. Ahora bien, si una determinada SICAV es verdaderamente una institución colectiva, la cuestión de su tributación al $1 \%$ es un problema de política fiscal y no de fraude ${ }^{28}$.

Con todo, el hecho de que no se exija el número mínimo de accionistas a las "SICAV índice" cuando se admiten a cotización ${ }^{29}$ y se permita -para la generalidad de estas entidades- la constitución de compartimentos diferentes, con 20 accionistas bajo un único contrato constitutivo, dificulta la posible declaración de conflicto en la aplicación de la norma. Piénsese que esta opción permite a los grandes patrimonios cumplir más fácilmente el requisito de difusión de capital, sin verse estorbados en sus decisiones por la entrada de inversores pequeños y medianos en compartimentos diferenciados, respecto de los que existe independencia absoluta en cuanto a responsabilidad, política de inversión o emisión de acciones dentro de la SICAV ${ }^{30}$.

\footnotetext{
24 Vid. arts. 9 de la Ley 35/2003, de 4 de noviembre, de Instituciones de Inversión Colectiva y 6 del Real Decreto 1082/2012, de 13 de julio, que aprueba el Reglamento de desarrollo de la citada Ley.

${ }^{25}$ Fuente: CNMV. (https://www.cnmv.es/DocPortal/Estadisticas/IIC/Estadisticas_IIC_2014_1T.pdf)

26 Ramos Muñoz, D. Y Ruiz Almendral, V.: "Los problemas tributarios de las Sociedades de Inversión en Capital Variable (SICAV)", op. cit., p. 28. Según el art. 15.1 de la Ley 58/2003, de 17 de diciembre, General Tributaria, "se entenderá que existe conflicto en la aplicación de la norma tributaria cuando se evite total o parcialmente la realización del hecho imponible o se minore la base o la deuda tributaria mediante actos o negocios en los que concurran las siguientes circunstancias: a) que individualmente considerados o en su conjunto, sean notoriamente artificiosos o impropios para la consecución del resultado obtenido; b) que de su utilización no resulten efectos jurídicos o económicos relevantes, distintos del ahorro fiscal y de los efectos que se hubieran obtenido con los actos o negocios usuales o propios".

${ }^{27}$ De La Torre Díaz, F.: "SICAV: una reforma necesaria", op. cit.

${ }^{28}$ De La Torre, F.: ¿Hacienda somos todos? Impuestos y fraude en España, Debate, 2014

${ }^{29}$ Vid. Art. 79 del Real Decreto 1082/2012, de 13 de julio, que aprueba el Reglamento de desarrollo de la Ley 35/2003, de instituciones de inversión colectiva .

${ }^{30}$ Vid. art. 6 y 80 del citado Real Decreto 1082/2012, de 13 de julio,.
} 
Luque, Víctor; Luque, Miguel Ángel, A vueltas con la SICAV. Reflexiones críticas desde el punto de vista económico-tributario.

La circunstancia que se está analizando también tiene otras implicaciones que afectarían al objeto social de este tipo de entidades -la inversión-, ya que una gran cantidad de ellas funcionan como "remansadoras" de rentas y de efectivo. Por ejemplo, es habitual que transcurran muchos meses sin que la sociedad haya realizado 'inversión' alguna. De esta forma, las SICAV permiten un diferimiento de la tributación mientras se mantenga la inversión en la sociedad y no se repartan beneficios ${ }^{31}$. Como se ha afirmado, "diferir indefinidamente un impuesto constituye una enorme ventaja fiscal, sobre todo si el vehículo es propio y se puede hacer literalmente lo que se quiera" ${ }^{32}$. No obstante, esta característica del diferimiento en la tributación se ha visto extendida a todas las ganancias patrimoniales obtenidas como consecuencia de la transmisión de las acciones o participaciones en cualquier tipo de institución de inversión colectiva, así como al reembolso de estas últimas, cuando el importe obtenido se destine a la adquisición o suscripción de otras acciones o participaciones en instituciones de inversión colectiva ${ }^{33}$.

En línea con lo anterior, se ha denunciado que las grandes fortunas están utilizando esta figura para capitalizarse exponencialmente por medio de la reinversión de sus beneficios ${ }^{34}$. Un reflejo de esta situación se observa en el hecho de que del total de beneficios obtenidos en el 2013 (alrededor de 2.400 millones de euros), sólo se repartieron entre los accionistas 11 millones de euros, lo que apenas supone un $0,46 \%$ del total ${ }^{35}$. Si a este hecho se le añade que en numerosas comunidades autónomas se ha suprimido de facto el Impuesto sobre Sucesiones, con reducciones en la cuota del $99 \%{ }^{36}$, el diferimiento en la tributación (de las ganancias de patrimonio) podría alcanzar a más de una generación. De esta forma se incrementa la tendencia señalada por Piketty (2014) a la concentración de la riqueza y al consiguiente incremento de la desigualdad ${ }^{37}$. Circunstancia que no coadyuva precisamente a la finalidad redistributiva de la renta predicable del sistema tributario, especialmente cuando se trata de impuestos globales ${ }^{38}$.

Es más, hasta el tercer trimestre de 2010, sus verdaderos "propietarios" obtenían liquidez sin necesidad de vender las acciones, mediante un mecanismo artificioso consistente en reducciones de capital y devolución de las aportaciones en concepto de prima de emisión, eludiendo durante muchos

\footnotetext{
${ }^{31}$ Según los datos de la CNMV, la participación en el patrimonio de la tesorería ha aumentado más de un $53 \%$, desde 2010 a 2014 , pasando de suponer un $3,2 \%$ a un $4,9 \%$.

${ }^{32}$ De La Torre, F.: ¿Hacienda somos todos? Impuestos y fraude en España, op. cit. Este autor señala que "en otros países no se exige el requisito de cien partícipes, porque las SICAV sí imputan rentas o son transparentes".

33 Vid. Art. 94 de la Ley 36/2006, de 28 de noviembre, del IRPF.

${ }^{34}$ Rocafort, G.: SICAV, paraíso fiscal, Rambla, 2011, p. 74.

${ }^{35}$ Fuente: CNMV (https://www.cnmv.es/DocPortal/Estadisticas/IIC/Estadisticas_IIC_2014_1T.pdf)

${ }^{36}$ Vid. COMISIÓN DE EXPERTOS PARA LA REFORMA DEL SiSTEMA TRIBUTARIO ESPAÑOL (2014): Informe, p. 245.

37 Vid. Piketty, T. (2014): "The Dynamics of the capital/income ratio" en Capital in the Twenty-first Century. Harvard University Press.

${ }^{38}$ Vid. Calvo Ortega, R.: ¿Hay un Principio de Justicia Tributaria?, Aranzadi, 2012, p. 64.
} 
Luque, Víctor; Luque, Miguel Ángel, A vueltas con la SICAV. Reflexiones críticas desde el punto de vista económico-tributario.

años el pago que hubiera correspondido por el correspondiente reparto de beneficios, teniendo en cuenta que el régimen jurídico específico de estas sociedades permite la ampliación o reducción automática del capital, dentro de unos límites, sin necesidad de acuerdo por parte de la junta general de accionistas.

La LPGE para 2001 modificó este aspecto, con efectos desde el 2 de septiembre de 2010, estableciendo que cuando los supuestos de reducción de capital de estas entidades tuvieran por finalidad la devolución de aportaciones, su importe se calificaría como rendimiento de capital mobiliario ${ }^{39}$. Precisamente, a partir de esa fecha se produce un incremento sustancial en la recompra de acciones (2.794 millones $€$ en los ejercicios 2011 y 2012), en comparación con la cifra de distribución de beneficios, que en ese mismo período no alcanzó los 30 millones de euros ${ }^{40}$.

Por otro lado, como reconoce la CNMV, "la gran mayoría de las SICAV negocian sus títulos en el denominado Mercado Alternativo Bursátil" ${ }^{41}$. Este mercado exige a las empresas unos menores requisitos de información ${ }^{42}$-en comparación con las exigencias del mercado continuo- lo cual implica una menor transparencia, dificultando, en cierta forma, su control. Reflejo o consecuencia de esto es que de poco más de veinte empresas que operan en este mercado, tres de ellas ${ }^{43}$ han visto suspendida su cotización en los últimos tiempos. Además, con la exigencia de cotización bursátil existía la posibilidad de aplicar un test de liquidez efectiva o "material" a las acciones de la SICAV, pero con el sistema actual que presupone la liquidez de los títulos, en la medida en que la sociedad debe atender las órdenes de compra o venta de los mismos, dicho requisito se cumpliría, aunque la mayoría de los accionistas sean meros hombres de paja, lo que dificultaría en gran medida la apreciación de la existencia de un fraude de ley o conflicto en la aplicación de la norma tributaria $^{44}$.

\section{LA COMPETENCIA EN EL CONTROL DE LAS SICAV}

A diferencia del resto de sociedades y contribuyentes, el control de los requisitos exigidos para la aplicación del tipo de gravamen del $1 \%$ a las SICAV no lo realiza la Agencia Estatal de Administración Tributaria (AEAT), sino la

\footnotetext{
39 Artículo 65.1.c) de la Ley 39/2010, de 22 de diciembre, de Presupuestos Generales del Estado para 2011.

${ }^{40}$ Series estadísticas de la CNMV.

41 Tutorial de la CNMV sobre los "Fondos de inversión y las Sociedades de Inversión Colectiva", consultado el 26.09.2014 en: https://www.cnmv.es/TutorialFondos/ppal.htm

${ }^{42}$ Blanco, O. (2008). El mercado alternativo bursátil. Características y diferencias frente al mercado bursátil. Revista de derecho del mercado de valores, (2), 237-272. Vid. al respecto, los arts. 35.8, 53.8, 53 bis, de la Ley 24/1988, de 28 de julio, del Mercado de Valores.

${ }^{43}$ El último de los casos ha sido el de Gowex, empresa que se dedicaba principalmente a la instalación de puntos Wifi. Anteriormente Zinkia - productora de los dibujos Pokoyó- y Bodaclick -empresa dedicada a la comercialización de servicios relacionados con las bodas- sufrieron la suspensión.

${ }^{44}$ Ramos Muñoz, D. Y Ruiz Almendral, V.: "Los problemas tributarios de las Sociedades de Inversión en Capital Variable (SICAV)", op. cit. p. 18.
} 
Luque, Víctor; Luque, Miguel Ángel, A vueltas con la SICAV. Reflexiones críticas desde el punto de vista económico-tributario.

CNMV. Y ello es así desde que en 2004 se iniciaran inspecciones tributarias a centenares de SICAV por considerar que la mayor parte de los inversores eran ficticios y que tales sociedades debían haber pagado el $35 \%$ de sus beneficios, que era el tipo nominal entonces vigente en el IS, en lugar del $1 \%$. El fruto de tales inspecciones no se materializó en ingresos para las arcas públicas por dos motivos: primero, porque las Cortes Generales aprobaron, con el voto favorable de todos los grupos parlamentarios, salvo IU, la enmienda 81 que CIU presentó al Proyecto de Ley de "reformas en materia tributaria para el impulso a la productividad", que implicaba "quitar" la competencia del control del cumplimiento de los requisitos de las SICAV a la Inspección de Hacienda y trasladarla a la $\mathrm{CNMV}^{45}$, curiosamente cuando el propio vicepresidente de la CNMV era accionista de una SICAV ${ }^{46}$; y segundo, porque el Tribunal Económico-Administrativo Central (órgano administrativo, integrado en la Secretaría de Estado de Hacienda y Presupuestos) dictó una Resolución en 2007 considerando que dicha modificación tenía un carácter interpretativo y que, por tanto, debían anularse las liquidaciones giradas en ejercicios anteriores a la aprobación de la reforma, incluso las correspondientes al año $2000^{47}$.

Al respecto, se ha afirmado que es inédito en nuestro ordenamiento jurídico que otro órgano administrativo (en este caso, la CNMV) deba dar el placet previo para que la AEAT pueda comprobar la situación tributaria ${ }^{48}$. Todo ello, teniendo en cuenta que, como señalaba Hensel, "es un error científico creer en el invariable contenido de la ley con independencia del cuerpo organizativo encargado de aplicar la ley a la realidad"49. En este sentido, se ha advertido sobre el escaso número de iniciativas de la CNMV para suspender o revocar la autorización a las SICAV, despojándolas, en consecuencia, de su privilegiado

\footnotetext{
45 La citada enmienda finalmente aprobada, incluida en la Disposición adicional tercera de la Ley 23/2005, de 18 de noviembre, tenía el siguiente tenor literal: "Pérdida del régimen fiscal especial de las instituciones de inversión colectiva. Cuando se produzca el apartado uno del artículo 13 de la Ley 35/2003, de 4 de noviembre, de instituciones de inversión colectiva, el fondo o la sociedad de inversión pasarán a tributar en el Impuesto sobre Sociedades al tipo impositivo general o, en su caso, al previsto para las sociedades patrimoniales a partir del período impositivo que se iniciará el día siguiente a la adopción del acuerdo de revocación de la Comisión Nacional del Mercado de Valores”. Respecto al resultado de la votación, cfr. Diario de Sesiones del Congreso de los Diputados, año 2005, VIII legislatura, número 103, 30 de junio de 2005. En este sentido, Falcón Y Tella concluye que, tras esta reforma, es imposible que la Agencia tributaria se pronuncie sobre materias que son competencia de la CNMV, sobre todo si el pronunciamiento de la Agencia es contradictorio con el del órgano administrativo competente. Cfr. Falcón Y Tella, R.: "Las SIMCAV y el abuso de las facultades de auto-tutela de la Hacienda Pública", Quincena Fiscal, núm. 15/2005.

${ }^{46}$ Diagonal, n. ${ }^{\circ} 23,2011$, "SICAV, El Estado sigue favoreciendo a los más ricos y a la especulación financiera"; Rocafort Pérez, G.: SICAV, paraíso fiscal, op. cit., pp. 137 y 138.

${ }^{47}$ Resolución 2278/2007 del TEAC, de 22/11/2007, Vocalía Décima.

${ }^{48}$ Ramos Muñoz, D. Y Ruiz Almendral, V.: "Los problemas tributarios de las Sociedades de Inversión en Capital Variable (SICAV)", op. cit. p. 31.

${ }^{49}$ Hensel, A.: "La influencia del Derecho Tributario sobre la formación de los conceptos de Derecho Público", Hacienda Pública Española, núm. 22, 1973, p. 178.
} 
Luque, Víctor; Luque, Miguel Ángel, A vueltas con la SICAV. Reflexiones críticas desde el punto de vista económico-tributario.

régimen fiscal ${ }^{50}$. Incluso se ha dado el caso de una SICAV con un solo partícipe que no perdió su condición de institución de inversión colectiva ${ }^{51}$.

Por todo lo expuesto, compartimos la conclusión según la cual si lo que se quiere es "un régimen especial de grandes fortunas es preferible ser claro y transparente: hay que establecer requisitos que se cumplan y no requisitos que, como no se cumplen, se opta por quitarle su fiscalización a la Inspección de Hacienda" ${ }^{2}$.

\section{LA GESTIÓN DE LAS SICAV}

Seguidamente, realizamos una breve referencia al papel que desempeñan los grandes grupos financieros y entidades bancarias en la administración y gestión de los fondos invertidos en las SICAV, a través de las denominadas Entidades Gestoras. Para ello, se debe advertir que, en la práctica, el esquema básico asumido por la LIIC es el de una sociedad (la SICAV), compuesta por una serie de bienes y derechos que, a su vez, son invertidos en otros bienes y derechos, corriendo dicha tarea a cargo de un gestor profesional (Entidad Gestora). De esta forma y, con las debidas salvedades, se ha advertido que la relación SICAV-Sociedad Gestora se asemejaría a la relación Fondo-Sociedad Gestora, aunque en el caso de la primera conformada normalmente por un grupo familiar, lo que resaltaría el carácter de estas sociedades como meros instrumentos para la canalización de patrimonios ${ }^{53}$. Al respecto, se ha publicado que el Grupo Santander ha gestionado, a través de tres entidades, más de 500 SICAV, con un capital global superior a 4.000 millones $€$ y también tres entidades con depósitos de unas 700 SICAV. Del mismo modo, otras entidades relevantes en la gestión de SICAV y depósitos serían los bancos BBVA y Bankinter ${ }^{54}$.

Esta canalización del control del ahorro a través de los grandes grupos financieros se potencia aún más con los beneficios fiscales introducidos en la reciente reforma de la LIRPF, aplicables a determinados instrumentos de inversión ${ }^{55}$, lo que constituye un nuevo incentivo para el mantenimiento del

\footnotetext{
${ }^{50}$ Martín Seco, J.F.: Economía, mentiras y trampas, Península, 2012, p. 240. En este mismo sentido, De La Torre Díaz, F.: "SICAV: una reforma necesaria", op. cit.

${ }^{51}$ De La Torre, F.: ¿Hacienda somos todos? Impuestos y fraude en España, op. cit.

52 Ibidem.

53 Ramos Muñoz, D. Y Ruiz Almendral, V.: "Los problemas tributarios de las Sociedades de Inversión en Capital Variable (SICAV)", op. cit., p. 12.

${ }^{54}$ La gestora BBVA Patrimonios administra 291 sociedades con un capital de 3.007 millones de euros. Además, el BBVA tiene depósitos de 304 SICAV. Por su parte, Bankinter Gestión de Activos se encarga de 242 sociedades y 1.384 millones. Y el banco depositario Bankinter cuenta con 347 SICAV. Vid. Diagonal, n. ${ }^{\circ}$ 23, 2011, "SICAV, El Estado sigue favoreciendo a los más ricos y a la especulación financiera" y LUCAS, M.: "SICAV: analizando los datos públicos en la CNMV", 29-05-2012 (http://www.manuelalucas.com/?p=68).

55 Entre otros, los beneficios fiscales aplicables al plan de ahorro 5, los planes individuales de ahorro sistemático o la transmisión de elementos patrimoniales por mayores de 65 años a cambio de una renta vitalicia (art. Primero, apartados tres, veinticuatro, setenta y dos, ochenta y ocho, y noventa y siete de la Ley 26/2014, de 27 de noviembre, por el que se modifican la Ley 35/2006, de 28 de noviembre, del IRPF,
} 
Luque, Víctor; Luque, Miguel Ángel, A vueltas con la SICAV. Reflexiones críticas desde el punto de vista económico-tributario.

ahorro en determinados productos a largo plazo, con la consecuente concentración del control del ahorro privado en muy pocas manos. Por este y otros motivos, en el control de la riqueza financiera, la desigualdad es aún mayor de la ya de por sí elevadísima desigualdad en la propiedad de la riqueza ${ }^{56}$. Todo ello, teniendo en cuenta, en nuestra opinión, el riesgo que supone tal concentración para las decisiones democráticas y la soberanía nacional. ${ }^{57}$

No obstante, no es inusual que los grandes patrimonios familiares posean más de una SICAV y que, además, posean sociedades gestoras de SICAV ${ }^{58}$. En definitiva, nos encontraríamos con lo que se ha denominado "estructuras SICAV", entendidas como un conjunto de Sociedades con una "cabecera" o madre de la que cuelgan sus filiales a distintos niveles, y con una serie de sociedades gestoras y sociedades depositarias que gravitan a su alrededor ${ }^{59}$.

\section{EL RIESGO DE LA POSIBLE EMIGRACIÓN DE LOS CAPITALES}

En el contexto en el que nos encontramos, si bien es cierto que cualquier iniciativa que proponga un aumento en la tributación de este tipo de sociedades conlleva un riesgo de huida de los capitales invertidos a través de las SICAV a zonas de menor tributación ${ }^{60}$, por ejemplo a Luxemburgo ${ }^{61}$, no lo es menos que tal posibilidad no debe justificar que en un Estado de Derecho los beneficios tributarios se consigan contratando mariachis ni que el único beneficio fiscal que no fiscalice la Inspección sea precisamente el tipo del 1\% de la SICAV.

el texto refundido de la Ley del Impuesto sobre la Renta de No Residentes, aprobado por el Real Decreto Legislativo 5/2004, de 5 de marzo y otras normas tributarias.

56 Piketty, T. (2014): "Th estructure of inequality" en Capital in the Twenty-first Century. Harvard University Press.

${ }^{57}$ Un ejemplo de ello lo constituye la exigencia de modificación del artículo 135 de la Constitución, tramitada mediante el procedimiento de urgencia y aprobada en única lectura en 2011. Vid. De La Hucha Celador, F.: "La reforma constitucional de la Deuda Pública", REDF, núm. 153, 2012. Otro ejemplo de la presión de los mercados sobre las decisiones lo encontramos en el Informe para la Reforma Fiscal, donde textualmente se indica que la supresión de la dualidad impositiva en el IRPF, con una base general a tipo progresivo y una base del ahorro con tipo proporcional, "crearía serios conflictos con los mercados, que ya han asimilado y se encuentran cómodos con la fórmula impositiva actual y que vigilan muy de cerca nuestra política fiscal". COMISIÓN DE EXPERTOS PARA LA REFORMA DEL SISTEMA TRIBUTARIO ESPAÑOL (2014): Informe, p. 107.

${ }^{58}$ El art. 51.2 del Reglamento de Instituciones de Inversión Colectiva dispone que "la suma de las inversiones de las SICAV pertenecientes a un mismo grupo y de los fondos de inversión y SICAV gestionados por sociedades gestoras del mismo grupo en instrumentos financieros que incorporen derechos de voto sobre un emisor no podrá rebasar el $15 \%$ de los mismos, sin que en ningún caso pueda imputar la posibilidad de ejercer una influencia notable sobre dicho emisor".

${ }^{59}$ Rocafort, G.: SICAV, paraíso fiscal, op. cit., p. 80.

${ }^{60}$ Tras la subida del tipo de gravamen al $28 \%$ por parte de las Diputaciones Forales Vascas, a finales de 2009, en tan sólo unos meses, 142 de las 212 SICAVs que había en el País Vasco cambiaron su domicilio fiscal ("Huida masiva de SICAV en el País Vasco tras el aumento de la fiscalidad", http://www.cotizalia.com/noticias/huida-paisvasco-sicav-aumento-fiscalidad.html).

${ }^{61}$ Tal y como ha advertido la propia patronal de las SICAV (INVERCO). Las SIF luxemburguesas son instituciones de inversión colectiva propias de ese pequeño país. Tributan con un tipo aún más reducido del $0,01 \%$ - y son más flexibles que su homólogo español: permiten invertir en cualquier sector, esté o no cotizado en Bolsa. 
Luque, Víctor; Luque, Miguel Ángel, A vueltas con la SICAV. Reflexiones críticas desde el punto de vista económico-tributario.

Además, conviene precisar varias cuestiones en relación con la justificación de la posible emigración de los capitales para mantener el actual régimen privilegiado de las SICAV:

1) El hecho de que las SICAV tengan su domicilio en España no constituye ninguna garantía de que la inversión se mantenga en nuestro país ${ }^{62}$. De hecho, desde el año 2009, buena parte de la cartera patrimonial interior -especialmente la correspondiente a valores representativos de deuda pública española- se ha cambiado por la exterior -incrementándose los depósitos en entidades de crédito extranjeras y las inversiones en derivados extranjeros-, con tendencia a superarla próximamente $^{63}$. Como podemos ver en el gráfico 2.1, desde el 2009 hasta la fecha actual se ha producido una considerable caída de la participación en el conjunto del patrimonio de las SICAV de la cartera de inversiones internas mientras que han ganado peso la cartera de inversiones externas y la tesorería.

2) La supuesta huida podría paliarse exigiendo a los contribuyentes con sociedades de inversión en el extranjero que imputaran esas rentas conforme al régimen de transparencia fiscal internacional, introduciendo un régimen similar para las españolas, como se preveía inicialmente en los documentos preparativos de la reforma tributaria. No obstante, finalmente no se ha incorporado este aspecto para las SICAV constituidas y domiciliadas en la Unión Europea (p. ej. en Luxemburgo) ${ }^{64}$.

3) La pérdida de recaudación tributaria en caso de que realmente emigraran dichos capitales sería mínima para las arcas públicas españolas. Recuérdese que en los últimos dos ejercicios el conjunto de las SICAV solo pagaron poco más de 23 millones de euros, mientras que los beneficios antes de impuestos para este período fueron superiores a 3.752 millones de euros. Es más, teniendo en cuenta las nuevas obligaciones de declaración de bienes en el extranjero, el intercambio de información con otros países, se observa que, a veces, no es tan fácil deslocalizar los capitales y que, en todo caso, esa operación no está exenta de riesgo. De hecho, una política de mayor control tributario sobre estas entidades y sus accionistas, así como de persecución del fraude de ley, podrá aumentar la recaudación procedente de estas entidades aun descendiendo el número de las domiciliadas en España ${ }^{65}$.

4) En todo caso, lo que sí es cierto es que un aumento de la tributación de estas sociedades, manteniendo no obstante una situación beneficiosa aunque no simbólica y escandalosa, coadyuvaría a reforzar la conciencia fiscal de la mayoría de los ciudadanos -que piensan mayoritariamente que los impuestos son injustos, que los ricos pagan poco y que existe mucho

\footnotetext{
${ }^{62}$ De La Torre Díaz, F.: SICAV: Una reforma necesaria, op. cit.

${ }^{63} \mathrm{CNMV}$ (https://www.cnmv.es/DocPortal/Estadisticas/IIC/Estadisticas_IIC_2014_1T.pdf)

${ }^{64}$ Cfr. apartado 16 del art. 100 de la Ley 27/2014, de 27 de noviembre, del Impuesto sobre Sociedades.

$65 \mathrm{Si}$ se aumentara el impuesto a un exiguo $10 \%$, la recaudación aumentaría siempre y cuando la reducción del volumen de beneficio de las SICAV no fuera superior al $90 \%$.
} 
Luque, Víctor; Luque, Miguel Ángel, A vueltas con la SICAV. Reflexiones críticas desde el punto de vista económico-tributario.

fraude fiscal ${ }^{66}$-, lo que repercutiría en un aumento de la recaudación muy superior a esos 11,5 millones de euros anuales ${ }^{67}$.

5) Las inversiones de este tipo de sociedades no se destinan mayoritariamente a la financiación de la economía real ${ }^{68}$, sino que se materializan en el ámbito especulativo financiero, con lo que la emigración de tales capitales no repercutiría muy negativamente en la economía productiva. Piénsese, por ejemplo, que la eliminación del régimen privilegiado de las SICAV en el País Vasco no ha puesto en peligro su economía, que sigue siendo la comunidad autónoma con mayor renta per cápita de España.

6) Por último, conviene recordar que ningún principio constitucional exige un trato de favor a las grandes fortunas sobre la base a la posible huida de los capitales al extranjero, mientras que por el contrario, sí están recogidos en el artículo 31 de nuestra Constitución los principios de justicia tributaria, entre los que se encuentran el de igualdad, capacidad económica o progresividad, por ejemplo.

Llegados a este punto, podemos concluir diciendo que se ha perdido una nueva ocasión con la actual reforma tributaria para modificar el régimen jurídico de las SICAV, reconduciendo de nuevo el control de este tipo de sociedades a la AEAT, estableciendo un límite máximo personal en el accionariado del $5 \%{ }^{69}$, exigiendo en su caso distribución de resultados entre sus accionistas ${ }^{70}$ y apostando decididamente por aumentar su tributación en el marco de los países de la OCDE. Dado que el actual Gobierno ha preferido mantener el statu quo para estas entidades ${ }^{71}$, en aras de la coherencia, debería haber incorporado una propuesta para reformar el artículo 31 de la Constitución Española, ahora que está tan en boga esta cuestión, en los siguientes términos:

"Todos contribuirán al sostenimiento de los gastos públicos mediante un sistema tributario basado preferentemente en los impuestos indirectos y en las tasas. Se regirá por el principio de fomento de competitividad fiscal a la baja, para atraer a España empresas y capitales, beneficiando las inversiones, el ahorro y las instituciones financieras y los mercados. El principio de progresividad sólo será predicable de la tributación de las rentas del trabajo en el IRPF".

\footnotetext{
${ }^{66}$ CISS: Estudio 2953 Opinión pública y política fiscal (XXIX), http://datos.cis.es/pdf/Es2953mar_A.pdf

${ }^{67}$ Tributación media anual del conjunto de SICAV para el período 2009-2013. Fuente: CNMV

${ }^{68}$ Vid. la distribución del patrimonio social de la SICAV del año 2010 y del primer trimestre de 2011 publicado por la CNMV. Vid. el análisis sobre el trasvase de capitales extraídos del mercado de la producción de bienes y servicio al mundo de la simple intermediación de valores que representa la SICAV, así como la relación entre SICAV y fondos especulativos de alto riesgo, en Rocafort Pérez, G.: SICAV, paraíso fiscal, op. cit., pp. 127 a 131 y 163 a 165.

${ }^{69}$ Sobre la posible problemática de registro en la CNMV de SICAV domiciliadas en países de la Unión Europea que no reunieran el requisito de distribución de capital social que podría establecerse reglamentariamente, vid. SANZ GADEA: Impuesto sobre Sociedades (T I): comentarios, CEF, Madrid 2004, p. 76.

${ }^{70}$ De forma similar a lo establecido en el art. 6 de la Ley 11/2009, de 26 de octubre, por la que se regulan las Sociedades Anónimas de Inversión en el Mercado Inmobiliario.

${ }^{71}$ Más allá de introducir unos cuantos aspectos técnicos respecto a la tributación de las ganancias de patrimonio y reducciones de capital en el IRPF de los socios. Vid. art. Primero, apartados veintidós, sesenta y ochenta y cuatro de la Ley 26/2014, de 27 de noviembre.
} 
Luque, Víctor; Luque, Miguel Ángel, A vueltas con la SICAV. Reflexiones críticas desde el punto de vista económico-tributario.

La comprobación e investigación desarrollada en la aplicación de este sistema se centrará en los contribuyentes personas físicas con rentas medias y bajas y en las pequeñas y medianas empresas, en lugar de en las entidades financieras, las grandes corporaciones y las relevantes fortunas, para evitar la deslocalización empresarial, la huida de capitales y los conflictos con los mercados".

En todo caso, somos conscientes de la necesidad de avanzar hacia el diseño de un sistema tributario nacional en el contexto europeo, con una modulación de los principios de ambos ordenamientos y desde una posición de confluencia entre estos órdenes competenciales, "so pena de una pérdida de soberanía fiscal que derivara en un sistema inaceptable desde la perspectiva de los principios de justicia tributaria"72.

\footnotetext{
72 Fernández Marín, F. Y Fornieles Gil, A.: "Hacia un nuevo Sistema Tributario. Esbozo de los principios europeos y constitucionales", Estudios sobre el Sistema Tributario actual y la situación financiera del Sector Público. Homenaje al profesor Dr. D. Javier Lasarte Álvarez, IEF, 2014, p. 497.
} 
Luque, Víctor; Luque, Miguel Ángel, A vueltas con la SICAV. Reflexiones críticas desde el punto de vista económico-tributario.

\section{BIBLIOGRAFÍA}

Agencia Estatal de Administración Tributaria (2014): Informe Anual de Recaudación Tributaria.

Blanco, O. (2008): "El mercado alternativo bursátil. Características y diferencias frente al mercado bursátil", Revista de derecho del mercado de valores, (2), 237-272.

Calvo Ortega, R. (2012): ¿Hay un Principio de Justicia Tributaria?, Cívitas, Madrid.

CISS (2012): Estudio 2953, Opinión pública y política fiscal (XXIX).

Comisión de Expertos para la Reforma del Sistema Tributario Español (2014): Informe.

De la Hucha Celador, F. (2012): "La reforma constitucional de la Deuda Pública", Revista Española de Derecho Financiero, núm. 153, 13-18.

De La Torre Díaz, F. (2009): "SICAV: Una reforma necesaria", Dominio Público, 02-09-2009.

De La Torre Díaz, F. (2014): ¿Hacienda somos todos? Impuestos y fraude en España, Debate, Madrid.

Falcón y Tella, R. (2005): "Las SIMCAV y el abuso de las facultades de autotutela de la Hacienda Pública", Quincena Fiscal, núm. 15/2005, 5-10.

Fernández Marín, F. Y Fornieles Gil, A.: "Hacia un nuevo Sistema Tributario. Esbozo de los principios europeos y constitucionales", Estudios sobre el Sistema Tributario actual y la situación financiera del Sector Público. Homenaje al profesor Dr. D. Javier Lasarte Álvarez, IEF, 2014, p. 497.

Hensel, A.: "La influencia del Derecho Tributario sobre la formación de los conceptos de Derecho Público", Hacienda Pública Española, núm. 22, 1973, p. 178.

Lucas, M.: "SICAV: analizando los datos públicos en la CNMV", Manuel de Lucas, http://www. manuelalucas.com/?p=68.

Martín Seco, J.F. (2012): Economía, mentiras y trampas, Península, Barcelona.

Navarro Sanchís, J. (2010): "Sombras y penumbras en la declaración tributaria especial", Medidas y procedimientos contra el fraude fiscal. Hinojosa Torralvo, J.J. (dir.) Atelier, Barcelona, págs. 53-74.

Piketty, T. (2014). Capital in the Twenty-first Century. Harvard University Press, Cambridge.

Ramos Muñoz, D. Y Ruiz Almendral, V. (2006): "Los problemas tributarios de las Sociedades de Inversión en Capital Variable (SICAV)", Quincena Fiscal, no $18,9-34$.

Rocafort Pérez, G. (2011): SICAV, paraíso fiscal, Rambla, Elche.

Sanz Gadea (2004): Impuesto sobre Sociedades (T I): comentarios, CEF, Madrid, p. 76.

Tusquets Trías Bes, F.(2001): La Sociedad de Inversión Mobiliaria de Capital Variable (SIMCAV), Marcial Pons, Madrid, p. 64. 